\title{
BMJ Open Advanced image-supported lead placement in cardiac resynchronisation therapy: protocol for the multicentre, randomised controlled ADVISE trial and early economic evaluation
}

\author{
Philippe C Wouters (D) , ${ }^{1}$ Chris van Lieshout, ${ }^{2}$ Vincent F van Dijk, ${ }^{3}$ \\ Peter-Paul HM Delnoy, ${ }^{4}$ Pieter AFM Doevendans, ${ }^{1}$ Maarten J Cramer, ${ }^{1}$ \\ Geert WJ Frederix, ${ }^{2}$ Frebus J van Slochteren, ${ }^{1}$ Mathias Meine ${ }^{1}$
}

To cite: Wouters $\mathrm{PC}$, van Lieshout C, van Dijk VF, et al. Advanced image-supported lead placement in cardiac resynchronisation therapy: protocol for the multicentre, randomised controlled ADVISE trial and early economic evaluation. BMJ Open 2021;11:e054115. doi:10.1136/ bmjopen-2021-054115

- Prepublication history and additional supplemental material for this paper are available online. To view these files, please visit the journal online (http://dx.doi.org/10.1136/ bmjopen-2021-054115)

FJvS and MM contributed equally.

Received 06 June 2021 Accepted 07 October 2021

Check for updates

(C) Author(s) (or their employer(s)) 2021. Re-use permitted under CC BY-NC. No commercial re-use. See rights and permissions. Published by BMJ.

For numbered affiliations see end of article.

Correspondence to

Philippe C Wouters;

p.wouters@umcutrecht.nl

\section{ABSTRACT}

Introduction Achieving optimal placement of the left ventricular (LV) lead in cardiac resynchronisation therapy (CRT) is a prerequisite in order to achieve maximum clinical benefit, and is likely to help avoid non-response. Pacing outside scar tissue and targeting late activated segments may improve outcome. The present study will be the first randomised controlled trial to compare the efficacy of real-time image-guided LV lead delivery to conventional CRT implantation. In addition, to estimate the cost-effectiveness of targeted lead implantation, an early decision analytic model was developed, and described here.

Methods and analysis A multicentre, interventional, randomised, controlled trial will be conducted in a total of 130 patients with a class I or lla indication for CRT implantation. Patients will be stratified to ischaemic heart failure aetiology and 1:1 randomised to either empirical lead placement or live image-guided lead placement. Ultimate lead location and echocardiographic assessment will be performed by core laboratories, blinded to treatment allocation and patient information. Late gadolinium enhancement cardiac magnetic resonance imaging (CMR) and CINE-CMR with featuretracking postprocessing software will be used to semi-automatically determine myocardial scar and late mechanical activation. The subsequent treatment file with optimal LV-lead positions will be fused with the fluoroscopy, resulting in live target-visualisation during the procedure. The primary endpoint is the difference in percentage of successfully targeted LV-lead location. Secondary endpoints are relative percentage reduction in indexed LV end-systolic volume, a hierarchical clinical endpoint, and quality of life. The early analytic model was developed using a Markov-model, consisting of seven mutually exclusive health states.

Ethics and dissemination The protocol was approved by the Medical Research Ethics Committee Utrecht (NL73416.041.20). All participants are required to provide written informed consent. Results will be submitted to peer-reviewed journals.

Trial registration number NCT05053568; Trial NL8666.
Strengths and limitations of this study

- Real-time visualisation of targets for left ventricular lead implantation allows for user-friendly and accurate guidance.

- Use of a specific model of the lateral wall with relatively small segments, which limits fortuitous intarget lead placement.

- Health Technology Assessment offers better understanding of potential economic benefits of targeted implantation.

- Cardiac MRI allows for observer independent image acquisition, but has relatively limited temporal resolution.

- The study is limited in power to detect differences in clinical outcome, including echocardiographic response.

\section{INTRODUCTION}

Chronic heart failure (HF) is a major global health concern with a 5-year mortality rate of about $50 \%$. In about one-third of these patients, HF is accompanied by left ventricular (LV) conduction delay (ie, QRSduration $\geq 130 \mathrm{~ms}$ ), which is a predictor for worse prognosis. ${ }^{12}$ Cardiac resynchronisation therapy (CRT) greatly reduces morbidity and mortality in these patients, but the extent of response is inconsistent and highly dependent on adequate LV lead placement (LVLP). In-scar LVLP greatly increases risk of cardiovascular death and HF-hospitalisation, ${ }^{3}$ whereas pacing in an area of late activation is likely to improve outcome. ${ }^{4-6}$ Moreover, a suboptimal lead position cannot be compensated by optimising device programming, ${ }^{7}$ rendering adequate LVLP arguably the cornerstone of this device therapy. 
Because the optimal location is highly variable and patient-specific, an individualised and targeted approach is often warranted. ${ }^{8}$ Previous research has demonstrated the benefits of image-guided lead delivery for improving clinical outcome. ${ }^{89}$ However, most studies did not allow for electrical guidance in the control group and allowed for only eight potential targets for lead deployment, thereby limiting the accuracy of lead deployment and increasing the odds of fortuitous 'in-target' lead placement. ${ }^{1011}$ Moreover, no large studies allowed for real-time visualisation of optimal targets, and most of the imageguided studies were not conducted in a true multicentre setting. As such, the current evidence for image-guided LVLP has remained relatively limited, and contemporary LVLP is still largely based on an empirical strategy. ${ }^{1}$

The present study protocol describes the first multicentre randomised controlled trial investigating advanced image supported lead placement in CRT (ADVISE). The primary aim of the study is to demonstrate the feasibility of reaching predefined segments through accurate image-guidance, using an 18-segment LV lateral wall model with live visual guidance during the implantation. The secondary objective is to investigate clinical efficacy by evaluating differences in 1 ) the extent of $L V$ reverse remodelling, 2) a hierarchical clinical endpoint and, 3) quality of life between both groups. Finally, a Health Technology Assessment will be conducted to determine the expected cost-effectiveness of a patient-tailored approach for targeted lead placement.

\section{METHODS AND ANALYSIS}

The ADVISE trial is a multicentre, randomised, controlled trial that is blinded to the patient and assessors of outcome (figure 1). Assessment of LV dimension, LV function and lead location will be performed by core laboratories. Patients will be stratified according to aetiology of $\mathrm{HF}$ in order to assure equal distribution of patients with ischaemic cardiomyopathy (ICM) and nonICM patients in both groups. All 130 patients will be 1:1 allocated to either image-guided or empirical LVLP using variable block-randomisation.

- Intervention group: live visualised, fluoroscopyfused, image-guided, lead placement on the basis of avoiding scar and targeting late mechanically activated segments.

- Control group: empirical standard-of-care lead placement, in line with current CRT implantation guidelines with electrical guiding using Q-LV sense. ${ }^{4}$

\section{Study population}

Patients are prospectively enrolled in at least three, and at most six, Dutch academic and peripheral centres. Consecutive patients eligible for CRT with a class I or IIa indication, with or without defibrillator function, according to the 2016 European Society of Cardiology Guidelines for the diagnosis and treatment of acute and chronic $\mathrm{HF}$

\section{Baseline assessments}

- Clinical evaluation (NYHA, ECG).

- Echocardiography and cardiac MRI.

- Two quality of life questionnaires.

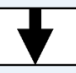

\section{MRI analysis and target allocation}

- Semi-automated analysis of scar and mechanical activation.

- Target allocation based on pre-specified decision-model.

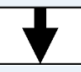

\section{1:1 variable block-randomization}
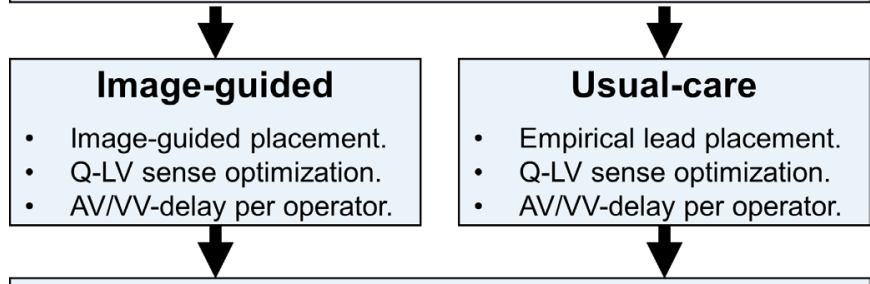

\section{6 months}

- Clinical evaluation (NYHA, ECG).

- Echocardiography.

- Two quality of life questionnaires.

\section{$12 \& 24$ months}

- Two quality of life questionnaires

- Clinical events (hospitalisation, LVAD, transplant, death)

Figure 1 Flow-chart presenting the course of the study. Legend: $\mathrm{AV}$, atrioventricular; LVAD, left ventricle assist device; MRI, magnetic resonance imaging; NYHA, New York Heart Association; VV, interventricular.

are considered. In addition, some additional criteria for study participation apply (box 1 ).

\section{Overview of assessments}

Prior to device implantation, all patients will undergo echocardiographic examination and cardiac magnetic resonance imaging (CMR). CMR feature-tracking (CMR-FT) analyses will be performed in both study groups, after which optimal LV-lead location will be determined. Randomisation will occur after targets for lead deployment have been defined, after which targets cannot be altered. All patients will receive two quality of life questionnaires (EQ-5D-5L and Kansas City Cardiomyopathy Questionnaire) at four timepoints: before implantation and at 6 months, 12 months and 24 months after implantation. A 12-lead ECG will be performed before, directly after and 6 months after implantation. During the procedure, various LV-paced effects will be measured. Ultimate lead location will be assessed through registration of the 18-segment LV lateral wall model onto the LAO40 and RAO30 fluoroscopy images, similar to the method described by Singh and colleagues. ${ }^{12} \mathrm{~A}$ global schedule of all assessments is summarised (figure 2). 


\section{Box 1 ADVISE inclusion and exclusion criteria}

\section{Inclusion criteria}

- Heart failure with LVEF $\leq 35 \%$.

NYHA class II, III or IV (ambulatory).

- Optimal medical treatment that is tolerable.

- LBBB with QRS $\geq 130 \mathrm{~ms}$, or non-LBBB with $\mathrm{QRS} \geq 150 \mathrm{~ms}$.

\section{Exclusion criteria}

- Age $<18$ years or incapacitated adult.

- Contraindication for CMR (gadolinium; contrast agents; metal).

- Atrial fibrillation; either permanent or during CMR.

- Severe renal insufficiency (GFR $<30 \mathrm{~mL} / \mathrm{min} / 1.73 \mathrm{~m}^{2}$ ).

- Participation in other potentially confounding trials. Legend: ADVISE, advanced image supported lead placement in CRT; CMR, cardiac magnetic resonance imaging; GFR, glomerular filtration rate; LBBB, left bundle branch block; NYHA, New York Heart Association.

\section{CMR analysis and target allocation}

Clinical standard short axis CINE acquisitions with a minimum of 25 frames per R-R interval, at max $8 \mathrm{~mm}$ slice thickness and no slice gap and late gadolinium enhancement (LGE) acquisitions at max $8 \mathrm{~mm}$ slice thickness will

\begin{tabular}{|c|c|c|c|c|c|}
\hline \multirow[b]{2}{*}{ Time point } & \multirow{2}{*}{$\begin{array}{l}\text { Enrolment } \\
-3 \text { months }\end{array}$} & \multirow{2}{*}{$\begin{array}{l}\text { Allocation } \\
\text { Day } 0\end{array}$} & \multicolumn{2}{|c|}{ Post-allocation } & \multirow{2}{*}{$\begin{array}{l}\text { Close-out } \\
24 \text { months }\end{array}$} \\
\hline & & & Day 3 & 6 months & \\
\hline \multicolumn{6}{|l|}{ ENROLMENT } \\
\hline Screening & $x$ & & & & \\
\hline Informed consent & $\mathrm{x}$ & & & & \\
\hline Cardiac MRI & $\mathrm{x}$ & & & & \\
\hline Echocardiography & $x$ & & & $\mathrm{x}$ & \\
\hline Electrocardiogram & $x$ & & $x$ & $x$ & \\
\hline NYHA class & $\mathrm{x}$ & & & $\mathrm{x}$ & \\
\hline Questionnaires & $x$ & & & $x$ & $x$ \\
\hline Target allocation & $\mathrm{x}$ & & & & \\
\hline Treatment allocation & & $x$ & & & \\
\hline \multicolumn{6}{|l|}{ INTERVENTIONS } \\
\hline Image-guided & & $\longrightarrow$ & & & \\
\hline Empirical & & $\longrightarrow$ & & & \\
\hline \multicolumn{6}{|l|}{ ASSESMENTS } \\
\hline LV-lead location & & $x$ & & & \\
\hline Procedural aspects a & & $x$ & & & \\
\hline Technical aspects & & $x$ & & & \\
\hline LVESVi- reduction & $x$ & & & $x$ & \\
\hline Resynchronisation ${ }^{b}$ & $x$ & & & $x$ & \\
\hline $\mathrm{QRS}_{\text {AREA }}$ & $x$ & & $x$ & $\mathrm{x}$ & \\
\hline Mortality & & $x$ & $x$ & $x$ & $x$ \\
\hline HF Hospitalisation & & $x$ & $x$ & $x$ & $x$ \\
\hline Adverse events & & $\mathrm{x}$ & $\mathrm{x}$ & $\mathrm{x}$ & $x$ \\
\hline
\end{tabular}

Figure 2 SPIRIT time schedule of enrolment, interventions and assessments for the ADVISE trial. ${ }^{\text {almplantation time, }}$ radiation exposure and electrode configurations. ${ }^{b}$ For example, indices of mechanical resynchronisation, such as apical rocking. Legend: ADVISE, advanced image supported lead placement in CRT; HF, heart failure; LVESVi, left ventricular end-systolic volume indexed to body surface area; SPIRIT, Standard Protocol Items: Recommendations for Interventional Trials. be performed in the participating hospitals. CMR scans may be acquired at most 6 months before implantation, in case of no (suspicion of) recent ischaemic events. Postprocessing will be performed in a centralised fashion using a dedicated software toolbox (CARTBox, CARTTech B.V., Utrecht, The Netherlands). The CARTBox analysis results in a treatment file, which will be used as an overlay with live fluoroscopy during the implantation procedure in the intervention group. Semi-automated and deep-learning assisted contouring CMR-FT analysis will be performed to quantify myocardial deformation and identify the tissue with the latest mechanical contraction. Scar transmurality will be identified based on the LGE acquisitions. Three dimensional maps of mechanical activation and scar transmurality are combined and used to define the optimal tissue (targets) for the LV-lead. Targets will then be allocated on the basis of a prespecified decision-model by two investigators, blinded to each other. Segments that contain myocardial scar will be disregarded, whereas segments with latest mechanical activation will be considered most appropriate. Because multiple regions may be deemed suitable, a maximum of three of the most suitable segments will be ranked and considered for implantation in that order of priority. In the case of initial disagreement, consensus will follow after discussion. Of note, the original unprocessed CMR will be available at the discretion of the implanting cardiologist, also in the control group.

\section{Echocardiography}

Transthoracic echocardiographic examinations will be performed at baseline and 6 months after CRT implantation at each participating centre. A standard local protocol used for strain-imaging in CRT candidates will be used, with special attention to high-quality images of the LV. To this end, each acquired image will include at least three separate beats, and LV strain images will be frame-rate optimised by using the narrowest sector width possible. LV volumes and function will be assessed using Simpson's bi-plane method. ${ }^{13}$ Mechanical dyssynchrony (eg, apical rocking) will be assessed as well. All examinations will be analysed by an echocardiography core laboratory using vendor-independent software.

\section{Randomization and blinding procedures}

After baseline assessments and subsequent identification of optimal targets for LVLP, computer-generated variable block 1:1 randomisation to either image-guided (intervention) or empirical (control) implantation will be performed (Castor EDC, Amsterdam the Netherlands). Randomisation will be stratified according to ischaemic or non-ischaemic HF aetiology. Study data will be collected, recorded, logged and managed in compliance with Good Clinical Practice guidelines. All study data are recorded in an electronic case report form (eCRF), where any changes in data entry are logged. All data entered, including perioperative data related to device implantation and optimisation, are collected and entered 
into the eCRF by either the coordinating investigator and/or research nurse. External data validation will be managed by a study monitor, designated by a contract research organisation. Both the patient and core laboratories assessing endpoint data (fluoroscopically determined LVLP and echocardiography) will be blinded to the intervention. After 6-month follow-up has been completed by all patients, unblinding is allowed. After 6 months, no observer-dependent endpoint data remains to be collected, and electrode reselection is allowed where indicated.

\section{Device implantation}

Implantation of CRT, unrestricted by manufacturer or the presence or absence of defibrillator, will occur under local anaesthesia and light intravenous sedation according to standard procedure. In the control group, LVLP will occur at discretion of the physician but in line with current guidelines using quadripolar LV leads (ie, based on an empirical strategy, guided by Q-LV sense). Q-LV sense is measured unipolar and defined as the time interval between QRS onset on the surface ECG and the maximum voltage change over time (ie, $\mathrm{dV} / \mathrm{dt}$ ), recorded on the ECG. The LV electrode with the longest Q-LV sense in combination with acceptable pacing threshold and without diaphragmatic stimulation will be selected. In the image-guided intervention group, two-dimensional fluoroscopic images are coregistered to the previously derived CARTBox treatment file from CMR postprocessing, and visualised in real-time in conjunction with the live fluoroscopy used during the implantation procedure (figure 3). The LV-lead will be deployed on the basis of the predefined target. Only when multiple electrodes are within the target region, electrode selection based on electrical properties (eg, Q-LV sense) is applied.

During the procedure, pacing capture thresholds, phrenic nerves stimulation, intrinsic electrical delay (ie, Q-LV sense) and various LV-paced effects (ie, LV-pace to $\mathrm{RV}$-sense and RV-pace to LV-sense) will be determined for each electrode of the quadripolar lead positions. When the ultimate lead position has been established, LAO40 and RAO30 fluoroscopic imaging will be performed to determine the exact final lead location. Final LV lead location will be determined by two investigators, blinded to treatment group and outcome of each other. LVLP will be determined through registration of

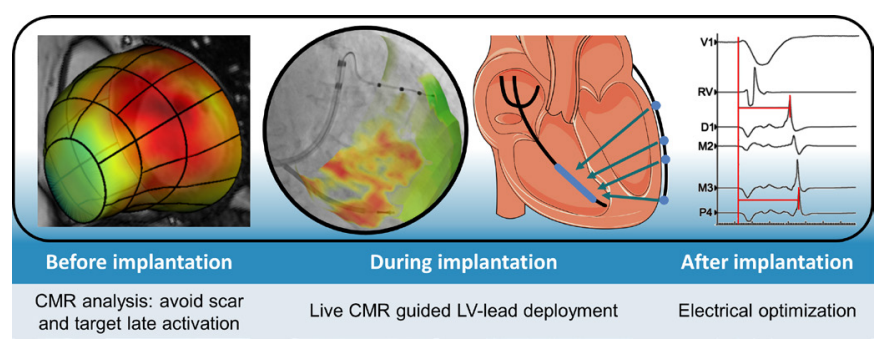

Figure 3 Workflow for advanced image-guided LV-lead placement. Adapted from Wouters et al. ${ }^{8}$ Legend: CMR, cardiac MRI; LV, left ventricular. the CMR-derived LV lateral wall model onto the LAO40 and RAO30 fluoroscopy images, similar to the method described by Singh and colleagues. ${ }^{12}$ Adverse events which are possibly related to CARTBox or the procedure, reported spontaneously by the subject or observed by the investigator or his staff, will be recorded in an electronic database.

\section{Endpoints}

The ability to achieve successful image-guidance will be based on differences in the percentage of within, adjacent or remote from the target(s) selected for lead placement. Here, adjacent segments include diagonal segments. Secondary outcomes are relative reduction in LV endsystolic volume indexed to body surface area (LVESVi), proportional difference in volumetric response $(\geq 15 \%$ LVESVi-reduction), differences in quality of life and differences in the CRT response score. The latter is a hierarchical clinical endpoint based on HF-hospitalisation and/or death within 12 months, relative LVESVi-change and change in New York Heart Association (NYHA) class (online supplemental file 1). ${ }^{14}$ Other outcome measures include the following: implantation procedure time, fluoroscopy time, contrast dose, device or procedure-related complications, change in QRS duration and QRS $_{\text {AREA }}$, indices of mechanical recoordination and LV-lead parameters (Q-LV sense, pacing threshold and phrenic stimulation). Finally, a Health Technology Assessment concerning the additional value of image-guided LVLP in terms of healthcare expenditure revolving HF care will be performed. This assessment will be based on a previously conducted early economic analysis, which is described in this article.

\section{Sample size}

When comparing image-guided and contemporary implantation of CRT, the proportional difference in within-target LVLP ranges between $6 \%$ and $30 \%$, and thus varies considerably. ${ }^{8}$ In contrast, ADVISE target segments approximately half the size of areas used in previous studies, rendering the chance of fortuitously successful in-target implantation in either study group much smaller.

We therefore hypothesised that image-guidance will result in a proportional difference in within-target LVLP of at least $27 \%$ when compared with empirical lead placement. In order to demonstrate this proportional difference using a two-sided Fisher exact test with $80 \%$ power and alpha $=0.05$, a total of 114 successfully implanted patients are needed.

Concerning the secondary endpoint of LV reverse remodelling, given an expected SD below 25\%, a significant difference in LVESVi reduction between both groups of at least 13\% can be detected in 116 patients. Accounting for failed implantations, loss to follow-up and incomplete (echocardiographic) data in about $10 \%$ of cases, total sample size necessary was set at 130 patients. 


\section{Statistical analysis}

An intention-to-treat analysis will be performed to assess LV-lead location and echocardiographic response. In echocardiographic non-responders where electrode reselection is feasible, transition of control patients towards the treatment group may occur after 6 months. To account for this potential cross-over, an additional per-protocol analysis may be performed with respect to long-term clinical endpoints and the Health Technology Assessment.

The primary endpoint concerning LV-lead location will be defined categorically as being within, adjacent or remote from the predefined target. A two-tailed Fisher exact test will be performed to assess differences in lead location between both groups. Because in principle, the effect of a targeted approach is considered to result in a unidirectional change in lead location, a one-tailed Fisher exact test may be performed as well.

Secondary endpoints will be analysed according to treatment allocation and lead location using Student's t-test and one-way analysis of variance, or Wilcoxon's rank sum test and Kruskal-Wallis wherever applicable. Finally, intraobserver and interobserver agreement of the echocardiography core laboratory analysis of LV reverse remodelling will be demonstrated by computing intraclass correlation coefficients in approximately 25 echocardiograms. A p-value $<0.05$ will be considered significant.

\section{Patient and public involvement}

Patients are part of our multidisciplinary consortium, both before and during the study, and are as such involved in the design and conduct of the study. The priority of the research question, patient communication, study logistics and methods of recruitment have been informed by discussions with patients representing our study population.

\section{ETHICS AND DISSEMINATION}

The ADVISE trial will be conducted according to the principles of the Helsinki Declaration II and Good Clinical Practice guidelines. The protocol has been written in accordance with the Standard Protocol Items: Recommendation For Interventional Trials (SPIRIT) checklist. ${ }^{15}$ The study protocol has been approved by the Medical Research Ethics Committee Utrecht (NL73416.041.20). All participants are required to provide written informed consent, prior to study procedures (online supplemental file 2). Patients are currently being enrolled, with the first patient included in February 2021. Results will be disseminated at various presentations and will be submitted to peer-reviewed journals.

\section{EARLY ECONOMIC EVALUATION}

To estimate the expected impact on cost and effects of image-guided LVLP in CRT, an early decision analytic model was developed using a Markov-model consisting of

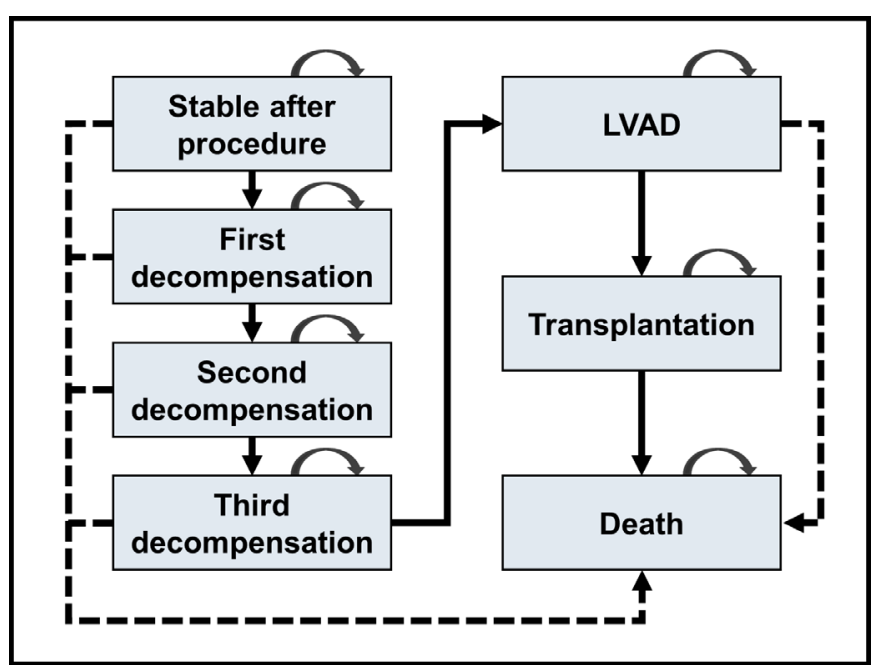

Figure 4 Seven 'health states' (squares) were defined. Patients either remain in their state during follow-up (inward arrows), or relocate towards the next sequential health state (uninterrupted arrows). Each transition is assigned its own probability of occurrence. When death occurs, other health states may be skipped (dashed arrows). Note that the assumption was made that post-CRT patients with $\leq 2$ decompensations will not receive LVAD or transplantation. Legend: CRT, cardiac resynchronisation therapy; LVAD, left ventricle assist device.

seven mutually exclusive health states (figure 4). These health states were identified in collaboration with clinical experts and based on available literature (online supplemental file 3). In brief, a group of 1000 individuals with HF were simulated, receiving either contemporary or image-guided LVLP. The analysis was performed from a societal perspective, including both direct healthcare costs and, where applicable, productivity losses due to absence from work. Model cycle length was 1 month, and model time horizon was 120 months. This model was developed in Microsoft Excel, V.2010/2016 (Microsoft, Redmond, WA, USA).

\section{Treatment of patients/structure of the model}

Patients with HF enter the model after the index CRT procedure (4:1 CRT-D vs CRT-P) where all patients are deemed to be in stable condition. After implantation, sequentially patients may 'transition' towards various 'health states', namely cardiac decompensation (at most three times), left ventricle assist device (LVAD) implantation, or heart transplant. Detailed overviews of healthcare provided for each of the health states are found in online supplemental file 2. Each health state is assigned a different probability of all-cause mortality.

\section{Input parameters}

Different sources were used to identify input parameters and parameter values. The majority of parameter values were retrieved from existing scientific literature. Where data were not publicly available, expert opinion and data from UMC Utrecht were used. Given the nature of this early analysis, no definitive data are currently available 


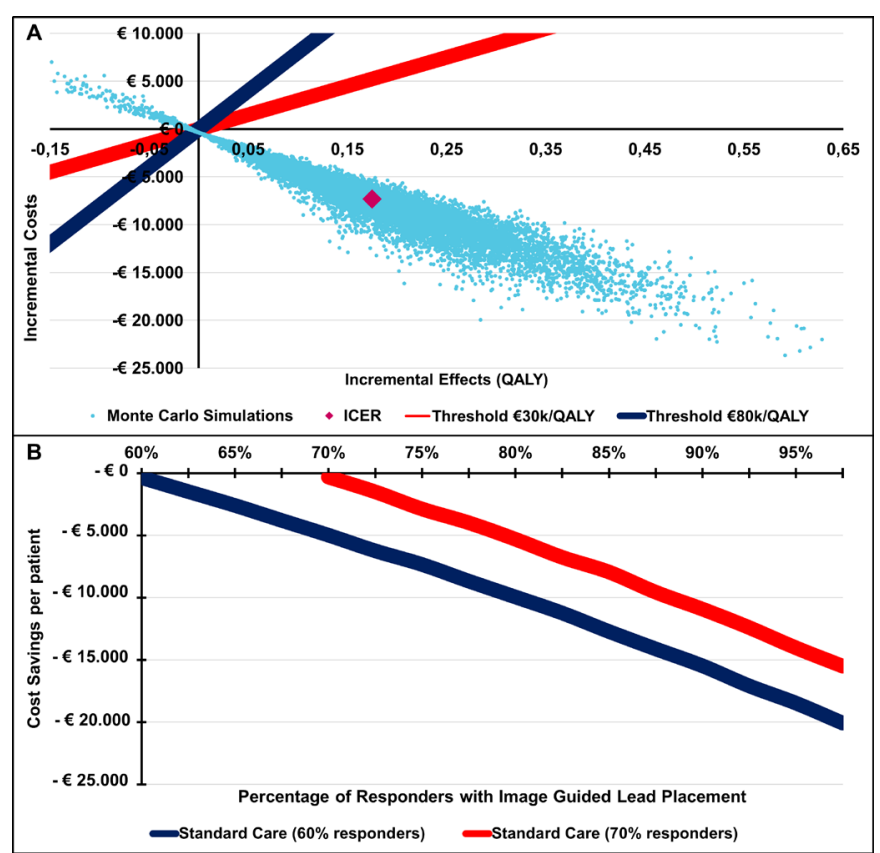

Figure 5 (A) Cost-effectiveness plane for image-guided lead placement. The graph shows the iterations (blue dots) in comparison to the cost-effectiveness thresholds for $€ 30$ 000/ QALY and €80 000/QALY (red and blue lines). (B) Potential cost savings with image-guided lead placement, based on the proportional difference in responders. Legend: ICER, incremental cost-effectiveness ratio ( $\triangle € / \triangle Q A L Y)$; QALY, quality adjusted life year.

that combines clinical effects and costs for image-guided LVLP. Input values for effects and costs were therefore estimated by experts.

For standard care, an assumed percentage of responders (LVESV-reduction $\geq 15 \%$ ) was set at $60 \%$ (17). For the additional effects of image-guided LVLP the percentage of responders was increased with steps of $2.5 \%$ to a maximum of $97.5 \%$. We also analysed the situation for when $70 \%$ of patients receiving standard care are responders. First decompensation probability, the arrow from stable to first decompensation in figure 4, was based on a weighted average for hospitalisation probabilities for responders and non-responders (18). Transfer probabilities between other health states were assumed to be equal between standard care and care with image-guided LVLP, and were based on clinical outcomes which were retrieved from literature (19-21). Most important index procedure complications were pneumothorax, lead dislocation, bleeding and pocket infection. Probabilities of these complications occurring were based on previous research conducted at the UMCU and were also assumed not to differ between image-guided LVLP and standard care (22).

\section{Cost-effectiveness estimation for image-guidance}

Based on 10000 Monte Carlo iterations in the probabilistic sensitivity analyses, figure $5 \mathrm{~A}$ shows the cost-effectiveness plane. Here, the Monte Carlo iterations are represented by the blue dots. Mean cost difference was found to be
$-€ 7.329$ (95\% CI: $-€ 15.760$ to $€ 323)$ and mean quality adjusted life year gain was 0.17 (95\% CI: -0.02 to 0.40$)$. The majority of iterations $(96 \%)$ resulted in cost saving and an incremental health gain for image-guided LVLP, as compared with standard care.

When the effectiveness of standard care is considered to be $70 \%$, and the effectiveness of CMR guided LVLP is varied between $70 \%$ and $95 \%$, the results shown in figure $5 \mathrm{~B}$ were found. Even at relatively small improvements in the proportion of responders, image-guided LVLP leads to cost savings ranging from $€ 317$ to $€ 20.069$.

\section{One-way sensitivity analysis}

In the one-way sensitivity analysis, we varied input parameter value with $-20 \%$ and $+20 \%$. This means the values of all parameters were altered one-by-one. By doing this for all model input parameters, the influence of each parameter on model outcome is demonstrated. The one-way sensitivity analysis showed that the parameters with the greatest influence on the outcomes of the economic evaluation were; (i) the percentage of responders for standard care, and (ii) the percentage of responders for care with image guided LVLP. This entails that changes in the value of these parameters will most likely change the outcomes of the economic evaluation the most.

\section{DISCUSSION}

\section{Electrical versus image-guided strategy}

Although the STARTER (Speckle Tracking Assisted Resynchronization Therapy for Electrode Region) and TARGET (Targeted Left Ventricular Lead Placement to Guide Cardiac Resynchronization Therapy) study demonstrated the benefit of an image-guided approach for LVLP, they were performed in a time where electrical guiding (using QLV-sense) was not yet routinely performed. ${ }^{10} 16$ However, QLV-guidance is nowadays readily available in many centres, and therefore, the results STARTER and TARGET cannot be directly extrapolated to current practice. ${ }^{417}$

It is therefore noteworthy that only one study carefully investigated both an electrically guided approach (using QLV-sense) and an image-guided approach in a direct comparison. ${ }^{18}$ Although Stephansen et al reported noninferiority of an electrical approach, we need to consider that these patients had typical left bundle branch block (LBBB) with an average QRS-duration of $169 \mathrm{~ms}$. This is in contrast to patients with non-LBBB morphology, where a QLV-guided approach fails to result in superior outcome when compared with contemporary lead placement. ${ }^{19}$ In these patients, an image-guided strategy appears to be more beneficial. ${ }^{16}$ Ultimately, electrically guided and mechanically guided approaches each have their own strengths and limitations, and both may have yet to reach their full potential. ${ }^{8}$ 


\section{Methods used for left ventricular lead placement}

Because in-scar pacing is associated with a six-fold increased risk for cardiovascular death or hospitalisation for $\mathrm{HF}$, avoiding in-scar pacing is of utmost importance. ${ }^{3}$ We therefore used CMR with LGE, which is considered the gold-standard for detection of myocardial scar and has a higher spatial resolution than ${ }^{82}$ Rubidium positron emission tomography. ${ }^{20}$ In contrast, the utility of strain imaging using echocardiography for detecting scar is poor with a sensitivity of only $33 \% .^{21}$

In addition to avoiding scar, feature tracking is performed on CMR CINE sequences in order to determine viable segments with late mechanical activation. Although CMR has lower temporal resolution than speckle-tracking echocardiography, its benefits include the ability to sequence the whole heart and the lack of need for adequate acoustic windows. In addition, strain analysis from CMR is subject to less bias and variability and can be done semi-automatically.

\section{Live fusion and target visualisation}

Regardless of the methods used, it is inevitable that there will always be patients in which a target cannot be reached. In particular, the variability and difficulty of reaching a predefined target is evidenced by the wide range of remote-from-target lead location, as reported in previous studies. ${ }^{8}$ Although venous access is undoubtedly a limiting factor, visualising targets for lead deployment during the procedure most likely enhances the proportion of optimally placed leads, since the implanter strives to implant the LV lead as close as possible to the target tissue in a patient specific fashion. Although the feasibility of live fusion has been demonstrated in two previous studies, ${ }^{22} 23$ they were limited by a small sample size and non-randomised design.

\section{Early economic analysis}

The analysis resulted in a robust model outcome for image-guided LVLP in CRT, demonstrating a mean cost savings of approximately $€ 7.000$ with simultaneous incremental health gain, relative to standard empirical LVLP. Although results are highly dependent on proportional differences in response, cost-savings are likely feasible even at relatively small clinical improvements. Because any decision analytic model is a simplified version of the actual healthcare pathway, definitive clinical effectiveness must be awaited from data gathered by the ADVISE trial. However, should the estimated mean cost savings hold, a meaningful improvement in cost-effectiveness can be realised. This may be especially valuable in low-to-middle income countries, where referral and implant rates are still relatively lacking. ${ }^{24}$

\section{Strengths and limitations}

Our study is primarily limited by its relatively small sample size, and as a consequence, lack of primary clinical endpoint with sufficient power to detect differences in LVESVi-changes below $13 \%$ between both groups. Regardless, the present study is the first multicentre randomised controlled trial set out to investigate live CMR-guided LVLP in CRT, thereby providing data in a real-world setting. CMR is however less suitable for patients with prior device implantation due to magnetic field inhomogeneities, reducing image quality. Although CMR-FT has a lower temporal resolution when compared with speckle-tracking echocardiography, it may suffer from less noise and interobserver dependence. Moreover, our technique allows for gold-standard scar detection, accurate segmentation and live visualisation of suitable targets for lead deployment. Finaly, although fluoroscopybased determination of LVLP has limited reproducibility, simultaneous coregistration with our CMR-derived LV lateral wall model may improve its accuracy. ${ }^{25}$

\section{Future perspectives}

Previous studies were conducted without performing QLV-guidance in the control group, and were limited by using at most two recruiting centres. ${ }^{10} 16$ Moreover, to date, no studies utilised live image-guidance in a randomised controlled design. Should our study be able to detect more LV reverse remodelling and/or better clinical outcome, an important step has been set towards more widespread adoption of image-guided strategies for optimised LVLP in CRT.

\section{Author affiliations}

${ }^{1}$ Department of Cardiology, Universitair Medisch Centrum Utrecht, Utrecht, The Netherlands

${ }^{2}$ Department of Public Health, Healthcare Innovation \& Evaluation and Medical Humanities (PHM), Universitair Medisch Centrum Utrecht, Utrecht, The Netherlands ${ }^{3}$ Department of Cardiology, Sint Antonius Ziekenhuis, Nieuwegein, The Netherlands ${ }^{4}$ Department of Cardiology, Isala Zwolle, Zwolle, The Netherlands

Contributors PW was involved in the study design, drafting the manuscript, and acquisition, analysis and interpretation of data. FJS was responsible for design and execution of the technical and methodological aspects revolving image guidance. $\mathrm{CL}$ and GWJF were responsible for design and execution of the early economic analysis. VFD, P-PHMD and MM were involved in study design and data acquisition. PAFMD and MJC were involved in conception or design of the work. All authors provided critical revision and final approval of the manuscript, and are accountable for the work.

Funding This work was supported by ZonMW, grant number 404460098327.

Competing interests FJS is cofounder, chief technical officer and shareholder of CART-Tech B.V. MM and FJS are inventors and beneficiaries of a patent license arrangement between the University Medical Centre Utrecht and CART-Tech B.V. according to the rules of the University Medical Centre Utrecht.

\section{Patient consent for publication Not required.}

Provenance and peer review Not commissioned; externally peer reviewed.

Supplemental material This content has been supplied by the author(s). It has not been vetted by BMJ Publishing Group Limited (BMJ) and may not have been peer-reviewed. Any opinions or recommendations discussed are solely those of the author(s) and are not endorsed by BMJ. BMJ disclaims all liability and responsibility arising from any reliance placed on the content. Where the content includes any translated material, BMJ does not warrant the accuracy and reliability of the translations (including but not limited to local regulations, clinical guidelines, terminology, drug names and drug dosages), and is not responsible for any error and/or omissions arising from translation and adaptation or otherwise.

Open access This is an open access article distributed in accordance with the Creative Commons Attribution Non Commercial (CC BY-NC 4.0) license, which permits others to distribute, remix, adapt, build upon this work non-commercially, and license their derivative works on different terms, provided the original work is 
properly cited, appropriate credit is given, any changes made indicated, and the use is non-commercial. See: http://creativecommons.org/licenses/by-nc/4.0/.

\section{ORCID iD}

Philippe C Wouters http://orcid.org/0000-0002-3406-4083

\section{REFERENCES}

1 European Society of Cardiology (ESC), European Heart Rhythm Association (EHRA), Brignole M, et al. 2013 ESC guidelines on cardiac pacing and cardiac resynchronization therapy: the task force on cardiac pacing and resynchronization therapy of the European Society of cardiology (ESC). developed in collaboration with the European heart rhythm association (EHRA). Europace 2013;15:1070-118.

2 Hawkins NM, Petrie MC, MacDonald MR, et al. Selecting patients for cardiac resynchronization therapy: electrical or mechanical dyssynchrony? Eur Heart $J$ 2006;27:1270-81.

3 Leyva F, Foley PWX, Chalil S, et al. Cardiac resynchronization therapy guided by late gadolinium-enhancement cardiovascular magnetic resonance. J Cardiovasc Magn Reson 2011;13:29.

4 Gold MR, Birgersdotter-Green U, Singh JP, et al. The relationship between ventricular electrical delay and left ventricular remodelling with cardiac resynchronization therapy. Eur Heart J 2011;32:2516-24.

5 Khan FZ, Virdee MS, Read PA, et al. Effect of low-amplitude twodimensional radial strain at left ventricular pacing sites on response to cardiac resynchronization therapy. J Am Soc Echocardiogr 2010;23:1168-76.

6 Kydd AC, Khan FZ, Watson WD, et al. Prognostic benefit of optimum left ventricular lead position in cardiac resynchronization therapy: follow-up of the target study cohort (targeted left ventricular lead placement to guide cardiac resynchronization therapy). JACC Heart Fail 2014;2:205-12.

7 Bogaard MD, Doevendans PA, Leenders GE, et al. Can optimization of pacing settings compensate for a non-optimal left ventricular pacing site? Europace 2010;12:1262-9.

8 Wouters PC, Vernooy K, Cramer MJ, et al. Optimizing lead placement for pacing in dyssynchronous heart failure: the patient in the lead. Heart Rhythm 2021;18:1024-32.

9 Jin Y, Zhang Q, Mao J-L, et al. Image-guided left ventricular lead placement in cardiac resynchronization therapy for patients with heart failure: a meta-analysis. BMC Cardiovasc Disord 2015;15:36.

10 Khan FZ, Virdee MS, Palmer CR, et al. Targeted left ventricular lead placement to guide cardiac resynchronization therapy: the target study: a randomized, controlled trial. J Am Coll Cardiol 2012;59:1509-18.

11 Saba S, Marek J, Schwartzman D, et al. Echocardiography-guided left ventricular lead placement for cardiac resynchronization therapy: results of the speckle tracking assisted resynchronization therapy for electrode region trial. Circ Heart Fail 2013;6:427-34.

12 Singh JP, Klein HU, Huang DT, et al. Left ventricular lead position and clinical outcome in the multicenter automatic defibrillator implantation trial-cardiac resynchronization therapy (MADIT-CRT) trial. Circulation 2011;123:1159-66.
13 Schiller NB, Shah PM, Crawford M, et al. Recommendations for quantitation of the left ventricle by two-dimensional echocardiography. American Society of echocardiography Committee on Standards, Subcommittee on quantitation of two-dimensional Echocardiograms. J Am Soc Echocardiogr 1989;2:358-67.

14 Packer M. Development and evolution of a hierarchical clinical composite end point for the evaluation of drugs and devices for acute and chronic heart failure: a 20-year perspective. Circulation 2016;134:1664-78.

15 Chan A-W, Tetzlaff JM, Gøtzsche PC, et al. SPIRIT 2013 explanation and elaboration: guidance for protocols of clinical trials. BMJ 2013;346:e7586.

16 Marek JJ, Saba S, Onishi T, et al. Usefulness of echocardiographically guided left ventricular lead placement for cardiac resynchronization therapy in patients with intermediate QRS width and non-left bundle branch block morphology. Am J Cardiol 2014;113:107-16.

17 Gold MR, Leman RB, Wold N, et al. The effect of left ventricular electrical delay on the acute hemodynamic response with cardiac resynchronization therapy. $J$ Cardiovasc Electrophysiol 2014;25:624-30.

18 Stephansen C, Sommer A, Kronborg MB, et al. Electrically vs. imaging-guided left ventricular lead placement in cardiac resynchronization therapy: a randomized controlled trial. Europace 2019;21:1369-77.

19 Singh JP, Berger RD, Doshi RN, et al. Targeted left ventricular lead implantation strategy for Non-Left bundle branch block patients: the enhance crt study. JACC Clin Electrophysiol 2020;6:1171-81.

20 Hunold $\mathrm{P}$, Jakob $\mathrm{H}$, Erbel $\mathrm{R}$, et al. Accuracy of myocardial viability imaging by cardiac MRI and PET depending on left ventricular function. World J Cardiol 2018;10:110-8.

21 Bakos Z, Ostenfeld E, Markstad H, et al. A comparison between radial strain evaluation by speckle-tracking echocardiography and cardiac magnetic resonance imaging, for assessment of suitable segments for left ventricular lead placement in cardiac resynchronization therapy. Europace 2014;16:1779-86.

22 Behar JM, Mountney P, Toth D, et al. Real-Time X-MRIGuided Left Ventricular Lead Implantation for Targeted Delivery of Cardiac Resynchronization Therapy. JACC Clin Electrophysiol 2017;3:803-14.

23 Shetty AK, Duckett SG, Ginks MR, et al. Cardiac magnetic resonance-derived anatomy, scar, and dyssynchrony fused with fluoroscopy to guide LV lead placement in cardiac resynchronization therapy: a comparison with acute haemodynamic measures and echocardiographic reverse remodelling. Eur Heart J Cardiovasc Imaging 2013;14:692-9.

24 Dickstein K, Normand C, Auricchio A, et al. CRT Survey II: a European Society of cardiology survey of cardiac resynchronisation therapy in 11088 patients - who is doing what to whom and how? Eur J Heart Fail 2018;20:1039-51.

25 Sommer A, Kronborg MB, Nørgaard BL, et al. Left and right ventricular lead positions are imprecisely determined by fluoroscopy in cardiac resynchronization therapy: a comparison with cardiac computed tomography. Europace 2014;16:1334-41. 